\title{
Semantically Enabling the Global Geodynamics Project: Incorporating Feature-Based Annotations via XML Pointer Language (XPointer)
}

\author{
L. I. Lumb \\ Computing and Network Services \\ York University \\ 4700 Keele Street \\ Toronto, Ontario M3J 1P3 \\ ian@yorku.ca
}

\author{
J. I. Lederman, J. R. Freemantle \& K. D. Aldridge \\ Earth \& Space Science and Engineering \\ York University \\ 4700 Keele Street, Toronto, Ontario M3J 1P3 \\ \{jerusha, keith\}@yorku.ca \\ freemant@eol.esse.yorku.ca
}

\begin{abstract}
Earth Science Markup Language (ESML) is efficient and effective in representing scientific data in an XML-based formalism. However, features of the data being represented are not accounted for in ESML. Such features might derive from events, identifications, or some other source. In order to account for features in an ESML context, they are considered from the perspective of annotation. Although it is possible to extend ESML to incorporate feature-based annotations internally, there are complicating factors identified that apply to ESML and most XML dialects. Rather than pursue the ESML-extension approach, an external representation for feature-based annotations via XML Pointer Language (XPointer) is developed. In previous work, it has been shown that it is possible to extract relationships from ESML-based representations, and capture the results in the Resource Description Format (RDF). Application of this same requirement to XPointer-based annotations of ESML representations results in a revised semantic framework for the Global Geodynamics Project (GGP).
\end{abstract}

\section{Overview}

In [6], an XML-based data model was introduced for the Global Geodynamics Project (GGP, [3]). This model was based on the Earth Science Markup Language (ESML, [16]). In addition to possessing a number of highly desirable traits, ESML also allowed for the extraction of a Resource Description Format (RDF) representation via Gleaning Resource Descriptions from Dialects of Languages (GRDDL) [7]. In this third paper in the series, ${ }^{1}$ the role of

\footnotetext{
${ }^{1}$ Both of the previous papers took the perspective of the grid enabling the GGP. In this paper, the shift to semantic enablement more accurately reflects the emphasis of these investigations.
}

GGP log data is reconsidered. The desire to properly leverage the semantic value latent in the GGP log data provides the rationale for reconsideration ( $(2)$. Invasive, internally driven approaches are considered and dismissed (also in $\S 2)$. Instead, attention is given to a non-invasive, externally driven method based on annotation ( $(3)$. The combination of an emerging World Wide Web Consortium (W3C) standard for XPointer and early access technology for the same through the W3C's Amaya facilitates the investigation. The resulting revised semantic framework for the GGP $(\S 4)$ and conclusions ( $\$ 5$ ) close out the current effort.

\section{Motivation}

Recent efforts aimed at automatically generating knowledge representations for GGP are summarized visually in Fig. 1. Although the details are provided elsewhere [6, 7], the key aspects captured by the visual summary are:

- On a monthly basis for each station, GGP data is comprised of primary (gravity and atmospheric pressure), auxiliary (e.g., ground water measurements) and log data, respectively, contained within three files.

- Three ESML templates, one for each of the three types of GGP data, allow for the automated conversion into an XML-based representation. Critical to the conversion is the use of an XML-based schema for ESML and software that accomplishes the required processing.

- From the XML-based representation of GGP data relationships are automatically extracted. Critical to this second step is the use of eXtensible Stylesheet Language Transformations (XSLT) to facilitate the conversion and software that produces RDF representations via GRDDL-based processing. 


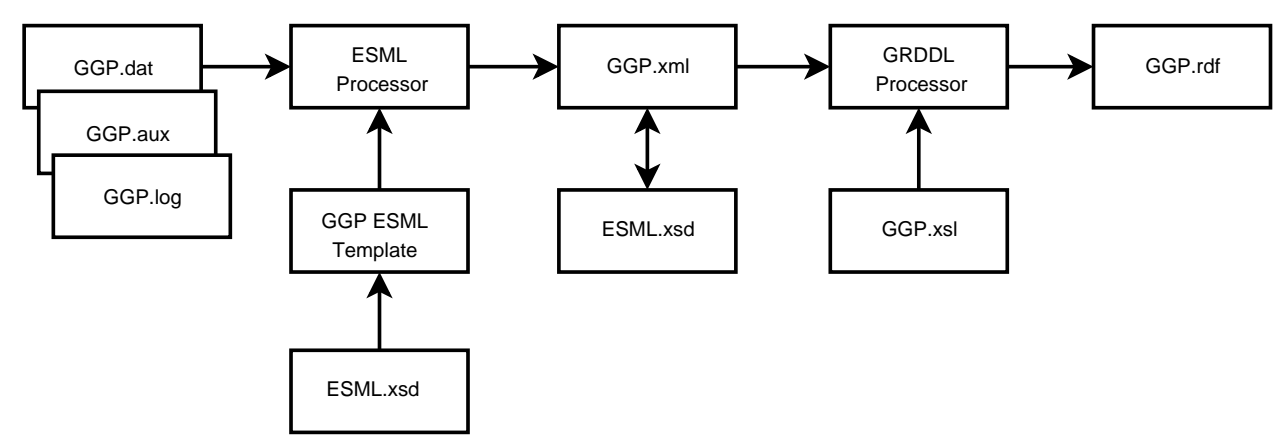

Figure 1. Schematic illustration of the progressive enhancement in semantic expressivity and richness of GGP data. Semi-structured ASCII data is represented in XML via ESML [6] and then RDF via GRDDL [7]. Because there are three input files, there are a corresponding number of XML and RDF representations.

By following through the progression implied by Fig. 1, it is clear that GGP log data is taken into consideration. However, on closer consideration of the data itself (Listing 1), it is clear that it remains a challenge to meaningfully relate GGP log data entries to either the GGP primary or auxiliary data. In the GGP case, the content of the log file is event-based and provided by the instrument operator. In some cases, the cause of the event is known and provided, but in others only the effect of some unknown event is detailed. Furthermore, some events are anticipated, whereas others are completely unexpected and possibly unexplainable. Date and time stamps are the only way to connect these event-based entries in GGP log data with the primary and auxiliary data.

\section{Listing 1. Example GGP log data ([18]).}

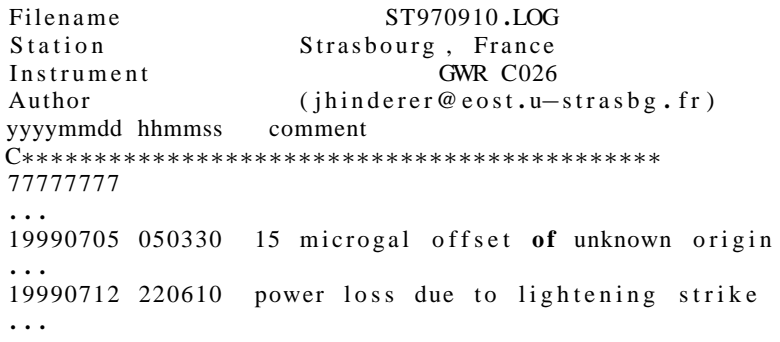

Although such date/time correlations are in principle possible, some effort will be required to make the correlation and then to somehow incorporate it into the overall representation illustrated at the outset (Fig. 1). The additional effort alluded to might involve, for example, use of XML Path Language (XPath) or XML Query Language (XQuery) to place the event. And although XSLT may allow XML-based representations to be manipulated, it is not clear that ESML can adequately incorporate such data into its representation. ESML is not alone in this regard, as it is anticipated that almost any XML-based dialect (includ- ing the Data Format Description Language, DFDL, standard, [19]) and frameworks analogous to [6] (e.g., [17]) will suffer from this same shortcoming. It may be possible to provide a clearer connection between events and the primary/auxiliary data once relationships have been introduced (the post GGP.xml portion of Fig. 1), but this will require significant manipulation of the various descriptions captured in RDF (via, e.g., XSLT). Ultimately, the difficulty being expressed is one of treating GGP log data as describing features of the GGP primary and auxiliary data.

Because the schema for ESML is readily available [16], there exists the possibility (in principle) of extending it. Given that ESML has been well established for a few years, and is presently in production use, schema extension has the disadvantage of deviating from vanilla ESML. And although such an extension may allow for GGP events to be represented, other challenges (e.g., correlating events and primary/auxiliary data) remain.

The types of events provided as examples in Listing 1 are of a point-in-time nature. It is conceivable that events could also have a duration. And in fact, it may be necessary to correlate events captured in one GGP log file with an event captured in another to adequately portray an event of some duration.

True to its XML heritage, ESML is based on the Document Object Model (DOM, [10]). Inherent with DOM is a container-based paradigm for document structure. In other words, documents are built by nesting elements according to rules established via the ESML schema. Examples using GGP log data suggest requirements for point-in-time and duration-of-time descriptions. Representational challenges aside, at least such feature do not violate the DOM inherent in XML.

Because interests are not limited to use cases derived from the GGP, it is important to anticipate situations where it might be necessary to violate the fundamental DOM be- 


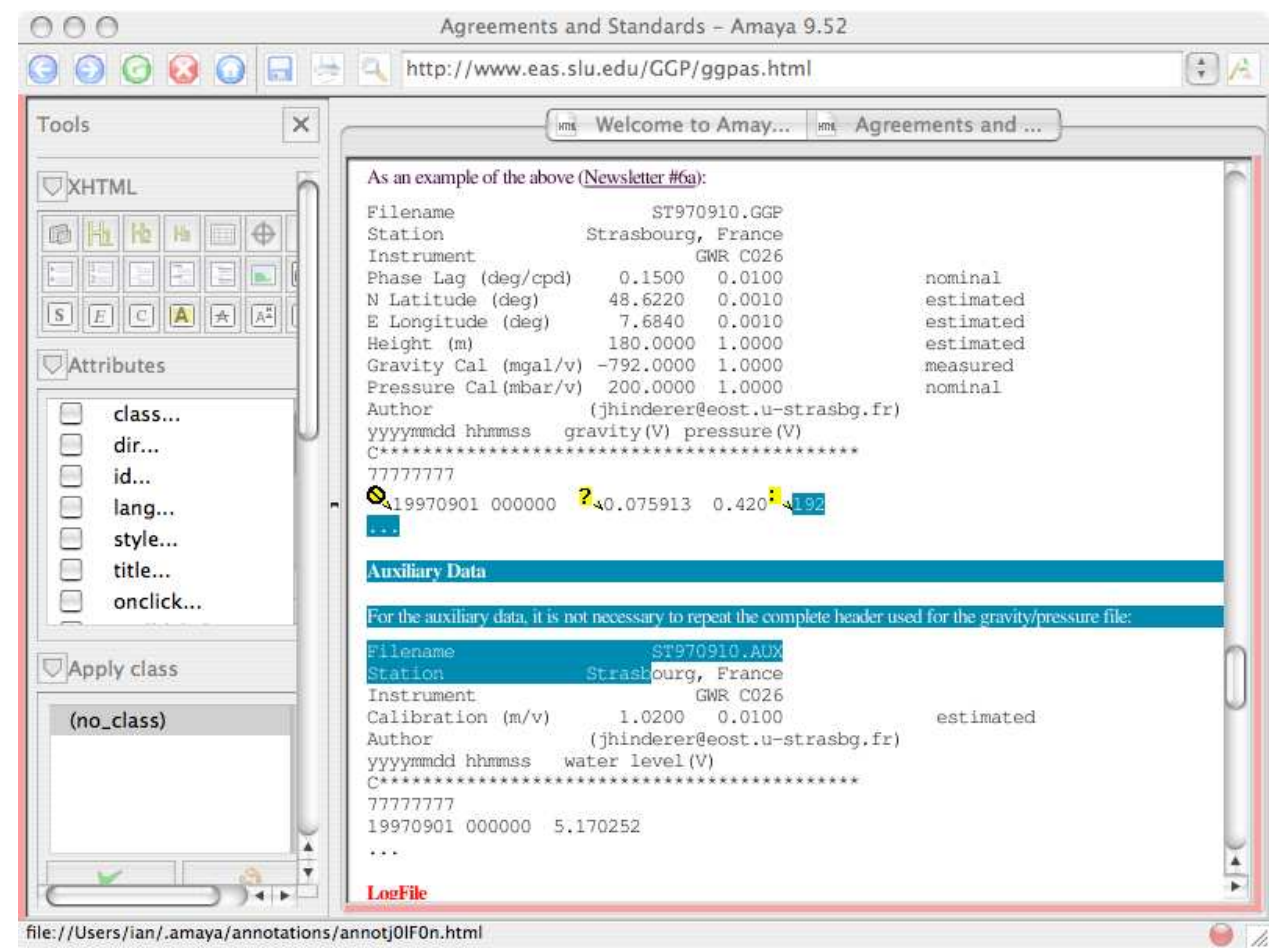

Figure 2. An identified URI fragment in Amaya. Note that this fragment spans a number of HTML elements making it a complex structure to annotate.

hind XML and XML Schema. Even cursory consideration of two-dimensional images provides for a compelling instance of such a violation. In such a case, ESML is DOM based, yet features may cross element boundaries. None of the possibilities for enhancing and/or extending ESML (including extending the ESML schema) can take into account such by-design violations. Again, true for ESML, this also applies to any XML-based representation.

\section{Representing Features via Annotation}

\subsection{Background}

Rather than proceed further with internally driven approaches (\$2), the present effort follows [9] in focusing on an externally driven approach based on annotation. From the archives of the W3C, Berners-Lee [1] provided one of the earliest definitions of annotation in a World Wide Web (WWW) context: "Annotation is the linking of a new commentary node to someone else's existing node. It is the essence of a collaborative hypertext." Not only well stated, Berners-Lee's definition emphasizes the highly collaborative aspect of annotation. A revised and expanded definition is also available from the $\mathrm{W} 3 \mathrm{C}$ via their Amaya software ${ }^{2}$

\footnotetext{
${ }^{2}$ Discussion of Amaya is deferred for the moment.
}

[20, Version 9.52]:

Annotations are comments, notes, explanations, or other types of external remarks that can be attached to a Web document or a selected part of the document. As they are external, it is possible to annotate any Web document independently, without needing to edit that document. From the technical point of view, annotations are usually seen as metadata, as they give additional information about an existing piece of data.

Because annotations convey additional information on an existing piece of data, they are metadata (i.e., data about data). However, to properly convey the value the annotations deliver, they are regarded as editorial metadata in the current context.

Armed with a definition and this level of familiarity, attention next turns to the appropriate standards and implementations that can be leveraged for annotation in the context of the GGP.

\subsection{Authoring Annotations with Amaya}

Before attention focuses on the details of how annotations are represented, consider the proof-of-concept illus- 
tration in Fig. 2. ${ }^{3}$ This figure demonstrates that the W3C's Amaya [20] is both a Web browser and development environment. ${ }^{4}$ In browsing mode, pre-existing annotations are indicated by use of yellow icons. The details of the icon convey the type of annotation (please see below). For the example provided in Fig. 2, on a mouse-over event the yellow icon with an enclosed colon, ":", illustrates via selected text an annotation of type Example. Furthermore, the selected content being annotated is a complex one, as it spans several HTML element boundaries.

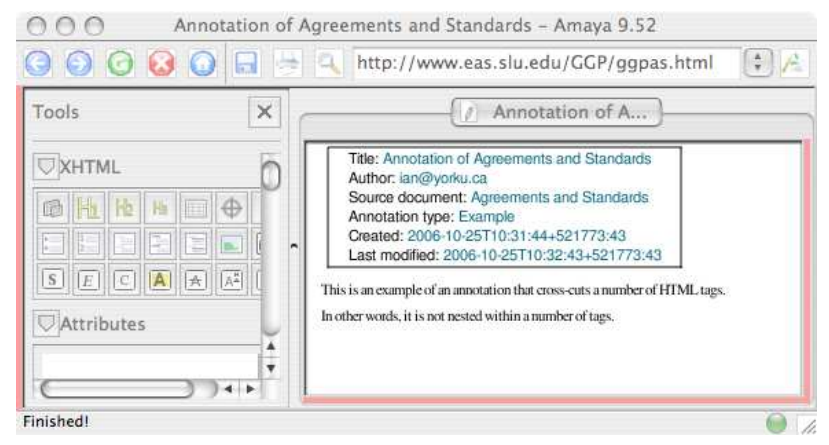

Figure 3. Annotation screen capture corresponding to the selection identified in Fig. 2.

Once the selection has been identified, use of Amaya's Annotations - Annotate Selection menu entries generates the screen capture illustrated in Fig. 3. Enclosed by the rectangle in the "Annotation of A..." panel is annotation metadata. Although most of this metadata is self-evident, it is worth noting that version 9.52 of Amaya has seven predefined types of annotations, namely Advice, Change, Comment, Example, Explanation, Question and SeeAlso. From this same panel of Fig. 3, it is clear that the type of annotation illustrated Fig. 2 is of type Example. Annotation type is the only example of annotation metadata that can be user-modified. Below the rectangle of annotation metadata appears the annotation itself. As the Tools panel at left in Fig. 3 implies, annotations can have rich context expressed via HTML. Amaya supports Reply $y^{5}$ and Post to Server functionalities. Both of these are powerful functionalities that will be explored in a future effort. Because Amaya has facilitated the authoring of an annotation, it is now possible to turn attention to how this annotation is

\footnotetext{
${ }^{3}$ The illustration is proof-of-concept in that use is made of an HTML representation of GGP data from [18]. A more complete demonstration would have GGP data represented in XML via ESML. There is, however, no loss of generality in the applicability of the approach.

${ }^{4}$ It is noteworthy that the W3C's aim in delivering Amaya is more around a demonstration platform for Web technologies rather than a fully fledged software product.

${ }^{5}$ An Amaya Reply is an annotation of an annotation.
}

actually represented.

\subsection{Representing Amaya's Annotations}

Another capability offered by Amaya's Annotations menu is Store selection as XPointer. The outcome of choosing this representation is provided in Listing 2. Before dissecting this listing in detail, the most striking observation is that an Amaya annotation is represented in RDF. Of course, this is not surprising as RDF is designed to make explicit relationships [15], and an annotation is editorial metadata related to a selection from a document. This RDF representation of annotations is highly appealing in the current context, as it will accelerate the incorporation of annotations into the emerging semantic framework for the GGP ([7] and Fig. 1). Before considering this integration in the next section ( $\S 4)$, it is important to expand on some of the details evident in Listing 2:

Namespace declarations In addition to the expected namespace declarations relating to RDF, there is a declaration specific to annotations.

http: //www.w3.org/2000/10/annotation-ns

is the Annotea Annotation Schema and is assigned in this listing to the variable $a$.

Resource declarations Following the namespace declarations are two annotation-specific resource declarations. Annotation is a class in the

http://www.w3.org/2000/10/annotation-ns

namespace, whereas Example is a class of annotation within the

http://www.w3.org/2000/10/annotationType

namespace.

Target resource for annotation Using the annotation namespace the resource being annotated, a GGP Web page [18], is identified. Amaya can be used to annotate any fraction of a document.

Context of the annotated resource The selection illustrated visually in Fig. 2 is referred to as an annotation context. This context is detailed via XPointer.

Annotation metadata and payload The listing closes with the annotation metadata that was illustrated graphically in Fig. 3. The only entry requiring elaboration is the annotation resource that specifies a local file, annotj01F0n.html. As can easily be intuited, this HTML file contains the editorial 
metadata that is the annotation itself; this is the text that appears below the rectangle in Fig. 3. Amaya randomly generates a fraction of this file name. The annotation payload is specified by a URL. This means that Amaya can also place such content on a remote server through the use of HTTP.

\section{Listing 2. A complex annotation represented in RDF by Amaya. Use is made of XPointer to detail the selection illustrated in Fig. 2.}

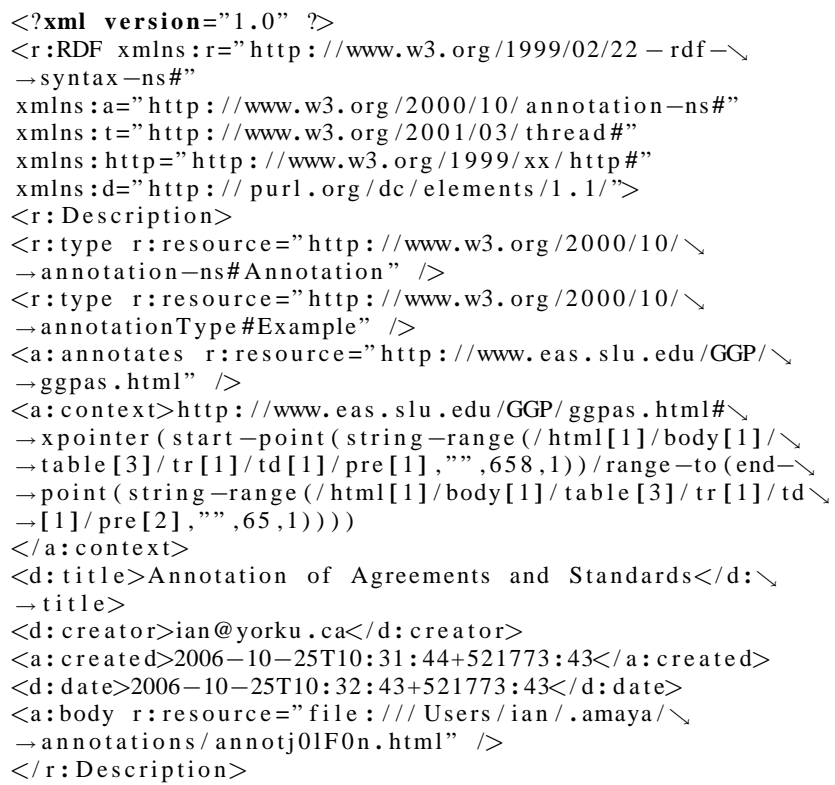

Because XPointer is the only significant addition to our existing semantic framework for the GGP [6,7], it is considered in more detail in the following section.

\subsection{Annotations via XPointer}

To accommodate annotation, the only required addition to the emerging semantic framework for the GGP [7] is XPointer [9]. The salient aspects of XPointer are as follows [10]:

- XPointer is an extension of XPath;

- XPointer allows for relative addressing, and this includes the ability to link to places (i.e., annotate selections) without anchors;

- XPointer is flexible and robust, and XPointer/XPath expressions often survive changes in the target document; ${ }^{6}$ and finally,

\footnotetext{
${ }^{6}$ See [2] for additional discussion on linking to living documents.
}

- XPointer can point to substrings in character data and to whole tree fragments.

Using the W3C's verbiage for XPointer [21], selections like that depicted in Fig. 2 are identified as fragments; thus XPointer is a fragment identifier for any URI (Universal Resource Identifier) reference. The standard for XPointer is comprised of four W3C specifications. Specifications for full XPath-based addressing [26], element-position addressing [23], and namespace binding [25], are each cast within a framework [24]. Examination of [26] reveals how XPointer numbers nodes and points, [22], to provide the relative addressing alluded to above. Also evident from this same specification, [26], is the identification of XPointer location types (start-point and end-point) and functions (string-range and range-to); this elaboration makes explicit XPointer's ability to serve as a fragment identifier for URIs while working within the constraints of the DOM [10] for XML.

The key XPointer specification, [26], is a W3C Working Draft. The other three specifications, [23], [24] and [25] are each W3C Recommendations. Despite the workin-progress nature of the primary specification, XPointer is progressing well along the path towards standardization.

Amaya abstracts away complexities inherent in XPointer. In other words, Amaya allows for early exposure, low-risk experience in annotating via XPointer. Given the progress to date and current status, it is anticipated that increasing investment in XPointer is warranted.

With annotations using XPointer via Amaya now considered, attention in the next section is placed on the same in the broader context of the emerging semantic framework for the GGP.

\section{Revised Semantic Framework for the GGP}

By recontextualizing GGP $\log$ data as annotations to GGP primary and auxiliary data, a revised semantic framework for the GGP is achieved (Fig. 4). From this figure it is evident that GGP log data, after user intervention involving Amaya, results in the annotation GGP_xml_annot\#1.rdf of GGP . xml $;^{7}$ the (red) arrow is intended to convey this annotation visually. Of course, there may be a number of annotations of the same GGP primary and auxiliary data. As indicated previously ( $\$ 3.3)$, Amaya is designed to handle multiple-annotation situations such as this, as is RDF (e.g., via RDF collections [15, Chapter 4]).

As before, the outcome achieved at this point is RDF representations for the GGP primary, auxiliary and log data. As suggested originally in [7, Fig. 5], it is maintained here

\footnotetext{
data.
} 


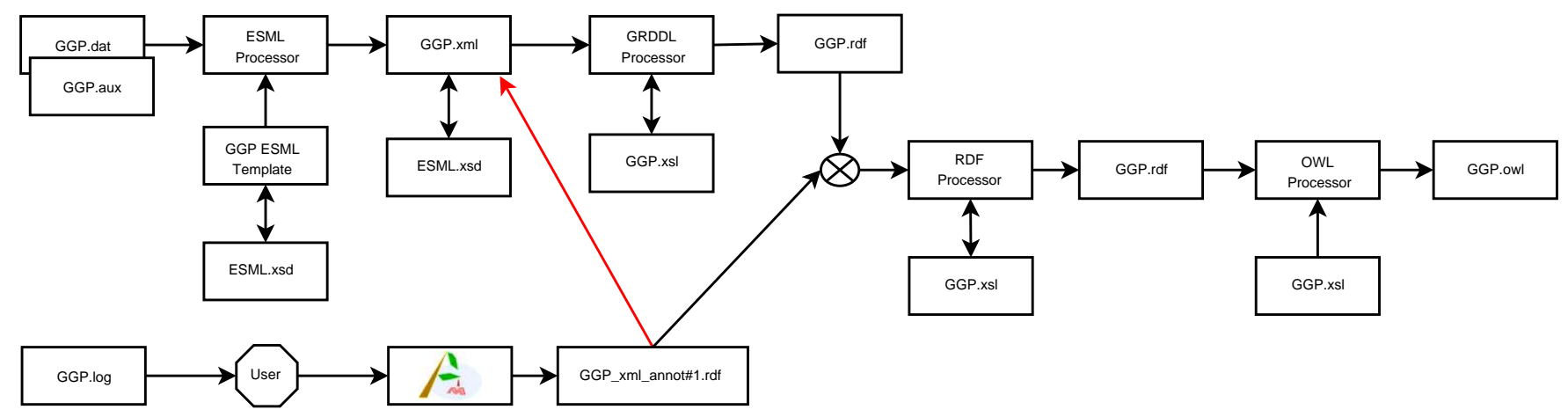

Figure 4. With the notable exception of the annotative approach applied to GGP log data, this figure is an analogous to the schematic illustration provided in Fig. 1. Use of W3C's Amaya results in an annotation represented in RDF. The resulting RDF representations for all GGP data are combined via XSLT rules into an informal ontology.

that the early extraction of RDF from ESML via GRDDL is the most effective approach for extracting relationships inherent in the GGP primary and auxiliary data.

With the annotative approach introduced here for GGP $\log$ data, there is now a need to merge the RDF-represented annotations with the RDF representation for the GGP primary and auxiliary data. Again, this merging (schematically illustrated by the circled " $x$ " in Fig. 4) of RDF representations is also likely to make (iterative) use of XSLT. The annotated GGP data can then be transformed into Web Ontology Language (OWL, $[4,14]$ ). Because OWL is a semantically richer and more-expressive dialect of RDF, XSLT is again anticipated to be of use. In the simplest case, the "OWL Processor" of Fig. 4 could be an engine for executing XSLT.

It is important to note that the eventual outcome of the semantic enhancement depicted in Fig. 4 is an informal ontology [7, 8]. In contrast to formal ontologies, e.g., [13], informal ontologies such as this one for the GGP are constructed from the bottom up. Of course as production experience with ontologies continues to expand, there is an ever pressing need to integrate ontologies [5]: “... we're in the throes of shifting from a need to emphasize ontology development, to a need to emphasize ontology integration." Thus it is anticipated that informal and formal ontologies also need to address the integration requirement.

Identified at the outset [6], one of the primary scientific objectives was to address the challenge of temporally and/or spatially aligning GGP data:

The requirement to correlate data, in time and space, is currently a very manual process that requires geodynamicists and seismologists to specify temporal (e.g., a period of time, an event in time) and/or spatial (e.g., global, regional, spe- cific instruments) details to allow for further analysis.

With the revised semantic framework for the GGP presented here, this scientific objective is well addressed. And because the framework is ultimately an XML-based representation, scientists have at their disposal various tools and utilities to develop and execute queries, plus temporally and/or spatially align and assemble on demand data sets for further analysis. As the semantic abilities of these tools and utilities improve, the extracted RDF and OWL representations can be increasingly leveraged. Ultimately, this will allow for natural-language queries in scientifically meaningful terms, e.g.:

Provide GGP data from all stations within a $10^{\circ}$ band of $45^{\circ} \mathrm{N}$ latitude for one year starting January 1,2006 . The data should have no gaps lasting longer than 4 hours.

With emerging support for semantically rich and expressive queries, it is clear that GGP data will find utility in broader contexts, e.g., the study of earthquakes and tsunamis [7].

\section{Conclusions}

Motivated by the desire to better extract the semantic value latent in GGP log data, the present effort has resulted in a revised semantic framework for the GGP. The revision is based on regarding GGP log data from the perspective of annotations. Thus the editorial metadata evident in GGP $\log$ data is appropriately related to the primary and auxiliary data from the GGP. Unlike invasive, internally directed solutions such as ESML schema extension, the approach based on annotation is non-invasive and externally directed. 
In reality, annotation is more pervasive and extensive than might be imagined, and numerous everyday examples exist. Known to be of relevance since the earliest days of the WWW by its inventor, annotation is of increasing relevance today. On the standards front, the W3C is close to approving XPointer as its approach for annotation via URI fragment identification. An early demonstration implementation of XPointer is also available from the W3C through the Amaya software for Web browsing and development. ${ }^{8}$ A proof-of-concept application of Amaya to GGP data illustrated that GGP log data can be represented as an annotation in XPointer.

Because Amaya represents annotations in RDF, the resulting annotations based on GGP log data can be readily integrated into the semantic framework for the GGP. The resulting RDF representations for the primary and auxiliary GGP data, plus the annotation-in-RDF representation of GGP $\log$ data, can all be transformed into OWL. Thus this is a case of an informal ontology being created from the bottom up. Given the increasing availability of both formally and informally derived ontologies, progress is soon to be determined by the ability to integrate ontologies.

\section{Acknowledgements}

Aspects of this work have been financially supported by KDA's NSERC operating grant, and by Computing and Network Services, York University; their contributions and ongoing support are gratefully acknowledged. Also, the authors gratefully acknowledge constructive feedback from two anonymous referees.

\section{References}

[1] T. Berners-Lee. Annotation. http://www.w3.org/DesignIssues/Annotation.

[2] T. Berners-Lee. The problem of linking to living documents. http://www.w3.org/DesignIssues/LinkToLiving.html.

[3] D. Crossley, J. Hinderer, G. Casula, O. Francis, H.-T. Hsu, Y. Imanishi, G. Jentzsch, J. Kaarianen, J. Merriam, B. Meurers, J. Neumeyer, B. Richter, K. Shibuya, T. Sato, and T. van Dam. Network of superconducting gravimeters benefits a number of disciplines. Eos Trans. Am. Geophys. U., 80:121126, 1999.

[4] M. C. Daconta, L. J. Obrst, and K. T. Smith. The Semantic Web: A Guide to the Future of XML, Web Services, and Knowledge Management. Wiley Publishing Inc., 2003.

[5] I. Lumb. The Pressing Need to Integrate Ontologies. http://ianlumb.wordpress.com/2006/09/08/ontology_merge_blog/.

[6] I. Lumb and K. D. Aldridge. Grid-enabling the Global Geodynamics Project: The introduction of an XML-based data

\footnotetext{
${ }^{8} \mathrm{An}$ analogous capability is now available for the popular Mozilla Firefox Web browser [12] via an annotation plug-in [11].
}

model. In I. Kotsireas and D. Stacey, editors, Proceedings of The 19th International Symposium on High Performance Computing Systems and Applications, HPCS 2005, pages 216-222. The IEEE Computer Society, 2005.

[7] I. Lumb and K. D. Aldridge. Grid-Enabling the Global Geodynamics Project: Automatic RDF Extraction from the ESML Data Description and Representation via GRDDL. In R. G. Deupree and J. A. Adams, editors, 20th International Symposium on High-Performance Computing in an Advanced Collaborative Environment, HPCS 2006, pages 184-191. The IEEE Computer Society, 2006.

[8] L. I. Lumb and K. D. Aldridge. Towards an ontology for the Global Geodynamics Project: Automated extraction of resource descriptions from an XML-based data model. Eos Trans. AGU, 86(52), 2005. Fall Meet. Suppl., Abstract IN43A-0328.

[9] L. I. Lumb, J. I. Lederman, and K. D. Aldridge. Incorporating Feature-Based Annotations into Automatically Generated Knowledge Representations. Eos Trans. AGU, 87(52), 2006. Fall Meet. Suppl., Abstract IN43D-06.

[10] A. Møller and M. I. Schwartzbach. An Introduction to XML and Web Technologies. Addison-Wesley, 2006.

[11] Mozdev.org. Annozilla (Annotea on Mozilla). http://annozilla.mozdev.org/.

[12] Mozilla. Mozilla Firefox. http://www.mozilla.com/enUS/firefox/.

[13] National Aeronatuics and Space Administration. Semantic Web for Earth and Environmental Technology (SWEET). http://sweet.jpl.nasa.gov/.

[14] T. B. Passin. Explorer's Guide to the Semantic Web. Manning Publications Co., 2004.

[15] S. Powers. Practical RDF. O'Reilly \& Associates, 2003.

[16] R. Ramachandran, S. J. Graves, H. Conover, and K. Moe. Earth Science Markup Language (ESML): A solution for scientific data-application interoperability problem. Comp. \& Geosci., 30:117-124, 2004.

[17] K. H. Rose, S. Malaika, and R. J. Schloss. Virtual XML: A toolbox and use cases for the XML world view. IBM Systems Journal, 45(2):411-424, 2006.

[18] The Global Geodynamics Project. Global Geodynamics Project Agreements and Standards. http://www.eas.slu.edu/GGP/ggpas.html.

[19] M. Westhead, T. Wen, and R. Carroll. Describing Data on the Grid. In 4th International Workshop on Grid Computing, pages 134-140. IEEE Computer Society, IEEE Computer Society, 2003.

[20] World Wide Web Consortium. Amaya Home Page. http://www.w3.org/Amaya/.

[21] World Wide Web Consortium. XML Pointer Language (XPointer). http://www.w3.org/TR/xptr/.

[22] World Wide Web Consortium. XPointer Document Order. http://www.w3.org/TR/xptr-xpointer/\#document-order-sec.

[23] World Wide Web Consortium. XPointer element() Scheme. http://www.w3.org/TR/xptr-element/.

[24] World Wide Web Consortium. XPointer Framework. http://www.w3.org/TR/xptr-framework/.

[25] World Wide Web Consortium. XPointer xmlns() Scheme. http://www.w3.org/TR/xptr-xmlns/.

[26] World Wide Web Consortium. XPointer xpointer() Scheme. http://www.w3.org/TR/xptr-xpointer/. 